\#097 Alterações microestruturais de materiais restauradores sujeitos a bebidas gaseificadas

Nuno Miguel Garrido Santos Costa*, Ana Sofia Coelho, Eunice Virgínia Carrilho, Anabela Paula

Faculdade de Medicina da Universidade de Coimbra

Objetivos: O estudo tem como propósito analisar a variabilidade de características dentárias como a dureza, rugosidade e peso, em função da absorção de um material restaurador estético, quando sujeito a condições ambientais como a imersão em cerveja, em refrigerante gaseificado e água. Filtek Supreme XTETM (3M ESPE, St. Paul, MN, EUA), resina composta nanoparticulada, e Ketac $^{\mathrm{TM}}$ Universal Aplicap ${ }^{\mathrm{TM}}$, ionómero de vidro, são os materiais selecionados para a consecução do estudo. O método aplicado no estudo foi análise comparativa, utilizando dados qualitativos e quantitativos das características dos espécimes selecionados.

Materiais e métodos: Trinta espécimes paralelepípedos $(6 \mathrm{~mm}$ x $13 \mathrm{~mm}$ x $4 \mathrm{~mm}$ ) de cada material foram preparados, polidos e armazenados numa estufa a $37^{\circ} \mathrm{C}$ durante 336 horas (14 dias). Os espécimes foram divididos em 6 grupos $(n=60)$. Grupos 1, 2: armazenados em água (Penacova ${ }^{\circledR}$ ). Grupos 3, 4: em cerveja (Super Bock $^{\circledR}$ ). Grupos 5, 6: em refrigerante gaseificado (Coca Cola ${ }^{\circledR}$ ). Foi realizada uma primeira avaliação após 7 dias (168 horas); e uma segunda avaliação após 14 dias (336 horas). Em cada avaliação foi registada a rugosidade superficial num rugosímetro, o peso numa balança de precisão e a dureza num durómetro. Os dados foram registados em tabelas e transcritos para gráficos específicos. Foram utilizados métodos One-Way-ANOVA, Tukey e Bonferroni, com um nível de significância de 5\%, para a análise estatística.

Resultados: Para o Filtek Supreme XTETM houve uma diminuição considerável da microdureza em todos os grupos. Para o Ketac Universal Aplicap ${ }^{\text {TM }}$ houve uma grande diminuição da microdureza. Relativamente à rugosidade, o parâmetro da média de rugosidade ( $\mathrm{Ra}$ ), da profundidade de rugosidade única (Rz), de rugosidade da média quadrática da raiz (Rq) e de skweness (Rsk) apresentaram-se constantes para todos os grupos de Filtek Supreme XTETM. No entanto, os parâmetros Ra e Rq registaram um aumento significativo nos grupos de Ketac Universal Aplicap ${ }^{\mathrm{TM}}$, sujeitos à cerveja e Coca-cola ${ }^{\circledR}$ (grupo 3, 5). O contrário foi registado para o Rsk, onde houve uma diminuição significativa dos valores. O Rz não foi considerado para o Ketac Universal Aplicap ${ }^{\mathrm{TM}}$ devido à sua inviabilidade.

Conclusões: O Filtek Supreme XTETM apresenta maior resistência à erosão por bebidas gaseificadas e consegue manter as suas propriedades microestruturais, quando comparado com o Ketac Universal AplicapTM.

http://doi.org/10.24873/j.rpemd.2017.12.120

\#098 Preparação e caracterização ultramorfológica de superfícies de blocos de resina composta

Nuno Gonzalez Correia*, João Carlos Ramos, Alexandra Vinagre

Faculdade de Medicina da Universidade de Coimbra

Objetivos: Os objetivos deste trabalho foram a avaliação ultramorfológica da preparação de superfícies de resina composta usadas em restaurações indiretas por técnicas CAD/CAM e a determinação das espessuras das interfaces de cimentação de blocos de resina composta, de acordo com cinco métodos distintos.

Materiais e métodos: No protocolo laboratorial, foi utilizado um bloco de compósito, Brilliant Crios Coltene CAD/CAM, seccionado sequencialmente numa máquina de corte por disco diamantado. A primeira fase do estudo laboratorial visou o estudo da micromorfologia superficial dos discos após diferentes tratamentos de superfície, tendo-se definido 4 grupos (Grupo 1 - controlo; Grupo 2 - jateamento com óxido de alumínio, a 45. ${ }^{\circ}$ e 90. ; Grupo 3 - jateamento com óxido de alumínio, a $45 .^{\circ}$ e $90 .^{\circ}$, e sujeita a vibração ultrassónica em álcool; Grupo 4 - jateamento com óxido de alumínio, a $45 .^{\circ}$ e $90 .^{\circ}$, e preparada com ácido fosfórico a 36\%). Para a segunda fase do estudo laboratorial, utilizaram-se 10 discos jateados, sujeitos a cinco protocolos de cimentação (Grupo 1 - Brilliant EverGlow $^{\circledR}$; Grupo 2 - Brilliant EverGlow ${ }^{\circledR}$ com aplicação de ultrassons; Grupo 3 Brilliant EverGlow ${ }^{\circledR}$ aquecido; Grupo 4 - Brilliant EverGlow ${ }^{\circledR}$ Flow; Grupo 5 - Duo Cem ${ }^{\circledR}$ Sample Trans).

Resultados: Na primeira fase do estudo laboratorial, ficou comprovado que o jateamento com óxido de alumínio, leva a uma alteração do padrão de superfície. Após a aplicação da vibração ultrassónica e de ácido fosfórico a $36 \%$, ocorre uma uniformização do padrão de superfície. Na segunda fase do estudo laboratorial, obtiveram-se diferentes espessuras da interface, de acordo com o protocolo de cimentação: Grupo 1 -

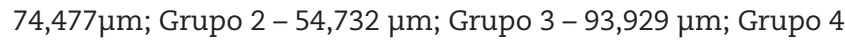
- 26,111 $\mu \mathrm{m}$; Grupo 5 - $45 \mu \mathrm{m}$.

Conclusões: A preparação da superfície interna dos blocos de resina com óxido de alumínio a $50 \mu \mathrm{m}$ é variável em função da orientação do jato, bem como da ação do ácido fosfórico ou vibração ultrassónica. As espessuras das interfaces de cimentação revelaram diferenças entre os protocolos estudados. http://doi.org/10.24873/j.rpemd.2017.12.121

\#099 Tecnologia CAD/CAM pelos Médicos Dentistas formados em Portugal

Pedro Cabral*, Filipe Miguel Araújo, Nuno Rosa, André Correia

CIIS - Centro de Investigação Interdisciplinar em Saúde, Instituto de Ciências da Saúde - Viseu

Objetivos: Identificar a presença e utilização da tecnologia CAD/CAM pelos Médicos Dentistas formados em Instituições de Ensino Superior Portuguesas.

Materiais e métodos: Estudo observacional transversal. Foi desenvolvido um questionário (adaptado de Tran, 2016) em suporte electrónico no programa informático Qualtrics ${ }^{\circledR}$ (Qualtrics Inc.). Este questionário foi enviado por e-mail a 265 Médicos Dentistas licenciados pela Universidade Católica Portuguesa (UCP) e 2347 sócios da Sociedade Portuguesa de Estomatologia e Medicina Dentária (SPEMD) Os e-mail foram enviados apenas uma vez. As questões elaboradas visavam obter informação acerca da aplicação, materiais utilizados, vantagens, desvantagens e limitações da utilização da tecnologia CAD/CAM em Medicina Dentária. O tempo médio de resposta era de 3-4 minutos.

Resultados: Foram obtidas 149 respostas [UCP - 68; SPEMD - 98). 61,1\% não utiliza nenhuma vertente dos siste- 
mas CAD/CAM, sobretudo devido aos elevados custos (49,5\%). Contudo, $61,1 \%$ estaria interessado em incluir esta tecnologia na sua atividade clínica. Das respostas obtidas, 36,6\% indicam que são utilizadores desta tecnologia, principalmente nas vertentes de CAD (22,86\%), CAM (22,86\%), e digitalização de impressões e/ou modelos em laboratório (21,2\%). As razões mais frequentes para utilização desta tecnologia foram: melhorar a qualidade $(30,00 \%)$ e uso de novos materiais dentários (27,5\%). Relativamente aos aspectos menos satisfatórios das restaurações obtidas por CAD/CAM foram referidos: estética (30,19\%); adaptação marginal $(15,1 \%)$ e oclusão (13,2\%). Os materiais dentários mais utilizados são as cerâmicas policristalinas $(42,0 \%)$ e as cerâmicas reforçadas (31,0\%). Relativamente à formação sobre sistemas CAD/ CAM, a maioria dos utilizadores são autodidatas (33,3\%) e/ ou recebeu formação das empresas; e cursos privados (31,8\%). 64,1\% considera que a formação recebida não foi suficiente. Clinicamente, $71,8 \%$ dos utilizadores sente que a presença de um sistema CAD/CAM no local onde exerce influencia as suas decisões clínicas. Por último, 94,7\% dos médicos dentistas que responderam aos inquéritos acham que o CAD/CAM terá um papel importante no futuro da Medicina Dentária.

Conclusões: Dentro das limitações deste estudo, particularmente relacionadas com o tamanho da amostra, verificou-se que apesar do interesse demonstrado, os custos elevados de aquisição da tecnologia CAD/CAM parece ser a principal barreira à sua utilização. De destacar a referência à falta de formação específica relevante nesta área.

http://doi.org/10.24873/j.rpemd.2017.12.122

\section{\#100 Adesão a dois cimentos de silicato de cálcio
usados em tratamentos pulpares regenerativos}

Joana Marques *, Rui Isidro Falacho, João Carlos Ramos, Francisco Caramelo, Alexandra Vinagre, Paulo Palma

Faculdade de Medicina da Universidade de Coimbra

Objetivos: Determinar as forças de adesão a dois cimentos de silicato de cálcio em dois tempos restauradores diferentes.

Materiais e métodos: 75 blocos de acrílico com cavidades foram preparados e distribuídos aleatoriamente por 5 grupos em função do cimento de silicato de cálcio, do timing da restauração e do material restaurador adesivo usado $(n=15)$. Grupos 1 e 2: as cavidades foram preenchidas com MTA (grupo 1) ou BiodentineTM (grupo 2) e somente após 7 dias de armazenamento em estufa a $37^{\circ} \mathrm{C}$ se procedeu à restauração adesiva através da aplicação de um sistema adesivo universal (Prime \& Bond activeTM) sobre o cimento de silicato de cálcio seguido de resina composta fluida (SDRTM). Grupos 3, 4 e 5: as cavidades foram preenchidas com MTA (grupos 3 e 4) ou BiodentineTM (grupo 5) e 12 minutos após a colocação do cimento de silicato de cálcio foram imediatamente efectuados os procedimentos restauradores adesivos (no grupo 3 com cimento de ionómero de vidro, GC Fugi IX GP, sem aplicação de qualquer sistema adesivo; nos grupos 4 e 5 o protocolo restaurador aplicado foi o mesmo descrito para os grupos 1 e 2). Os testes de adesão, em tensão de cisalhamen- to, foram realizados numa máquina de testes universal (Shimadzu) com uma velocidade de $0,5 \mathrm{~mm} / \mathrm{min}$ e os valores obtidos foram submetidos a tratamento estatístico utilizando o teste Dunn-Sidak como teste post-hoc, para um intervalo de confiança de $95 \%(<0,05)$. Foi efetuada avaliação dos padrões de fratura obtidos.

Resultados: O grupo 5 apresentou um valor médio de forças de adesão $(4,44 \mathrm{MPa})$ estatisticamente superior $<0,05)$ aos obtidos nos grupos 3 (1,14MPa) e 4 (1,33MPa), não se verificando diferenças na comparação com os grupos 3 e 4 entre si. Considerando a média dos valores de força de adesão obtidos nos grupos 1 (3,86MPa) e 2 (3,09MPa), não se verificaram diferenças entre os dois biomateriais. Considerando os dois intervalos temporais testados, o grupo 1 apresentou diferenças estatisticamente significativas $<0,05$ ) em relação ao grupo 4 , sem diferenças relativamente aos grupos 2 e 5 .

Conclusões: Considerando as limitações associadas ao presente estudo in vitro, os resultados obtidos sugerem que a aplicação de BiodentineTM poderá tornar viável a realização de procedimentos restauradores adesivos numa única sessão após o tratamento pulpar regenerativo.

http://doi.org/10.24873/j.rpemd.2017.12.123

\section{\#101 Avaliação da espessura de interfaces adesivas na colagem de fragmentos coronários}

Sofia Gaspar*, João Carlos Ramos, Rui Isidro Falacho, Ana Chambino, Ana Messias, Alexandra Vinagre

Faculdade de Medicina da Universidade de Coimbra

Objetivos: Avaliar a espessura de interfaces adesivas na colagem de fragmentos coronários, com recurso a uma resina composta aplicada de três formas diferentes: convencional, termo-modificada, e associada a vibração ultrassónica (estudo piloto).

Materiais e métodos: Quinze terceiros molares humanos foram divididos, aleatoriamente, por três grupos $(n=5)$. Cada dente foi seccionado, transversalmente, ao nível da coroa, de modo a obter um 'fragmento' coronal destacado. Os grupos foram definidos em função do método de colagem adesiva: grupo 1 - resina composta, à temperatura ambiente; grupo 2 - resina composta, à temperatura ambiente, com vibração ultrassónica; e grupo 3 - resina composta, aquecida a $50^{\circ} \mathrm{C}$. Os fragmentos foram colados utilizando um protótipo de controlo e medição da força, um sistema adesivo universal (Prime \& Bond ${ }^{\mathrm{TM}}$ Active, Dentsply DeTrey; LOT: 1702000441; VAL: 01-2019) e uma resina composta (Ceram $\mathrm{X}^{\circledR}$ duo, D3, Dentsply DeTrey; LOT: 0080; VAL: 06-2018). Após a colagem dos fragmentos, os espécimes foram seccionados e as interfaces adesivas foram observadas por microscopia eletrónica de varrimento e, posteriormente, medidas com recurso ao programa ImageJ (Image Processing and Analysis in Java). As comparações entre grupos foram efetuadas com recurso ao teste de Kruskal-Wallis, para um nível de significância de 5\%.

Resultados: A espessura média das interfaces adesivas para os 3 grupos foram (média $\pm d p$, em $\mu \mathrm{m}$ ): grupo 1 (194.7 \pm 88.3); grupo 2 (103.0 \pm 37.7$)$; grupo 3 (136.5 \pm 70.3). Apesar de 